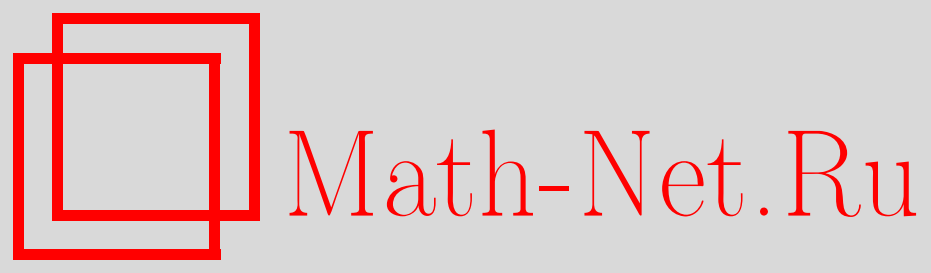

Л. Емельянов, Необычное определение конических сечений, Квант, 2021, номер 11, 2-4

DOI: https://doi.org/10.4213/kvant20211101

Использование Общероссийского математического портала Math-Net.Ru подразумевает, что вы прочитали и согласны с пользовательским соглашением http://www . mathnet.ru/rus/agreement

Параметры загрузки:

IP: 54.224.135.184

26 апреля 2023 г., 10:28:31

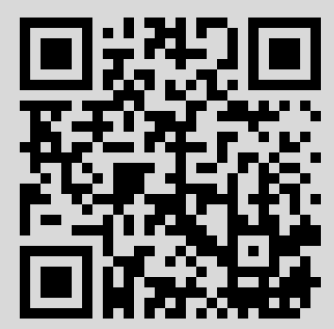




\section{Необычное определение конических сечений}

\section{Л.ЕМЕЛЬЯНОВ}

КАК ИЗВЕСТНО, ТРИ КОНИЧЕСLиш. сечения - эллипс, парабола и гипербола - могут быть определены с помощью прямой $d$ (директрисы) и точки $F$ (фокуса), на этой прямой не лежащей (см. рисунок 1 , а также «Калейдоскоп «Кванта» в этом номере). Кривая определяется

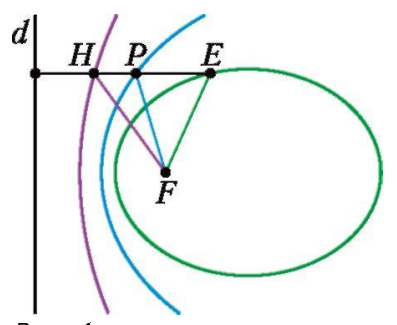

$$
\begin{aligned}
& \frac{F E}{\rho(E, d)}=e<1 \\
& \frac{F P}{\rho(P, d)}-e-1 \\
& \frac{F H}{\rho(H, d)}=e>1
\end{aligned}
$$

PnC. 1

как геометрическое место точек, для которых отношение расстояния до фокуса к расстоянию до директрисы есть заданная положительная константа $e$ (әксцентриситет). Если при этом $e<1$, мы получим әллипс, при $e=1$ выйдет парабола, а при $e>1$ - гипербола. Справедливости ради надо отметить и небольшой недостаток: в этой трактовке нет места окружности (для которой $e=0$ ).

Преимущество же этого подхода к определению конических сечений не только в однотипности, но и в «корректном» переходе через положение $e=1$. Для примера возьмем определение эллипса как множества точек с фиксированной суммой расстояний до фокусов. Если зафиксировать положение двух фокусов эллипса, а эксцентриситет устремить к 1, то в качестве «вырожденного эллипса» для $e=1$ мы получим отрезок, а не параболу. Аналогичные проблемы возникнут при переходе через положение $e=1$ «со стороны гиперболы»

DOI: https://doi.org/10.4213/kvant20211101
В этой статье предлагается альтернативный способ определения трех конических сечений некоторым однотипным подходом.

Рассмотрим фиксированные окружность $\omega$ радиуса $R$ и касательную к ней $l$. По прямой $l$ скользит отрезок $A B$ постоянной длины $2 a$, из концов которого проводятся вторые касательные к $\omega$, отличные от $l$. Найдем геометрическое место точек пересечения этих касательных.

На рисунке 2 представлены два варианта расположения касательных $C A$ и $C B$. Точка их пересечения $C$ и есть точка, принадлежащая искомому геометрическому месту.



a)

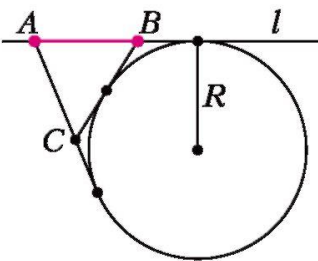

6)
PnC. 2

ГМТ будем задавать аналитически. Для определения уравнения ГМТ рассмотрим систему координат $x O y$ и поместим центр окружности в точку $Q(0 ;-R)$, прямая $l$ будет совпадать с осью $O x$, отрезок $A B$ длины $2 a$ будет скользить по $O x$. В качестве параметра, определяющего расположение отрезка, выберем координату его середины - $M(t ; 0)$. При этом параметр $t$ имеет право принимать любые действительные значения, а координаты концов отрезка будут таковы: $A(t-a ; 0)$ и $B(t+a ; 0)$ (рис. 3).

Из геометрических соображений ясно, что в зависимости от величины отноше- 
ния $a / R$, обозначим его через $e$, кривая, представляющая ГМТ, будет ограниченной или «разорвется» с уходом в бесконечHOCTb.

Составляя уравнения касательных к окружности из точек $A$ и $B$ и нахо-

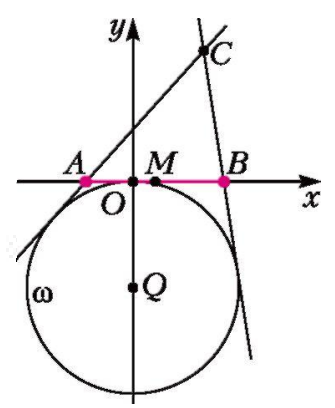

PUC. 3 дя их точку пересечения $C$, мы получим следующее параметрическое представление нашего ГМТ:

$$
x=\frac{2 R^{2} t}{t^{2}+R^{2}-a^{2}, y} \frac{2 R\left(a^{2} \cdot t^{2}\right)}{t^{2}+R^{2}-\vartheta^{2}} .
$$

Упражнение 1. Проделайте соответствующие выкладки, в результате которых получаются формулы (1).

Чтобы понять тип кривой, исключим $t$ из уравнений (1). В результате получим следующее уравнение кривой второго порядка:

$$
\begin{array}{r}
R^{2} x^{2}+\left(R^{2}-a^{2}\right) y^{2}+2 R\left(R^{2}-2 a^{2}\right) y= \\
=4 R^{2} a^{2} .
\end{array}
$$

Упражнение 2. Выведите (2) из (1). При этом можно воспользоваться такой схемой:

1) Поделив одно уравнение на другое, выразите $a^{2}-t^{2}$ как линейную функцию от $t$

2) Получите из одного из уравнений явное выражение для $t$ через $x, y$ (и параметры $a$ и $R$ ).

3) Подставив выражение для $t$ в другое уравнение, получите (2).

Теперь разберем три случая.

1. Парабола $(a=R)$.

В этом случае уравнение (2) принимает вид

$$
y=\frac{1}{2 R} x^{2}-2 R
$$

Таким образом, мы получаем параболу с фокусом $F\left(0 ;-\frac{3}{2} R\right)$ и директрисой $d$, имеющей уравнение $y=\frac{\bar{j}}{2} R$ (рис.4).

2. Эллипс $(a<R)$.

Уравнение (2) имеет вид

$$
\frac{x^{2}}{p^{2}}+\frac{\left(y-y_{0}\right)^{2}}{q^{2}}=1 \text {. }
$$



Pnc. 4

где

$$
\begin{aligned}
& p \frac{R^{2}}{\sqrt{R^{2}-a^{2}}}, q=\frac{R^{3}}{R^{2}-a^{2}}, \\
& \text { अ॥ } \frac{R\left(R^{2}-2 a^{2}\right)}{R^{2}-a^{2}} .
\end{aligned}
$$

Фокусы эллипса имеют координаты

$F_{1}\left(0 ;-R \frac{2 a+R}{R+a}\right)$ всегда внутри $\omega$,

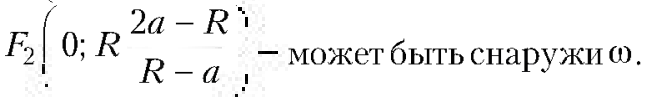

Эллипс (рис.5) вытянут по оси $\mathrm{Oy}$ и никогда не бывает окружностью.

Известно, что для эллипса, заданного каноническим уравнением $\frac{x^{2}}{a^{2}}+\frac{y^{2}}{b^{2}}=1$, где $0<b \leq a$, эксцентриситет опре- Рис. 5

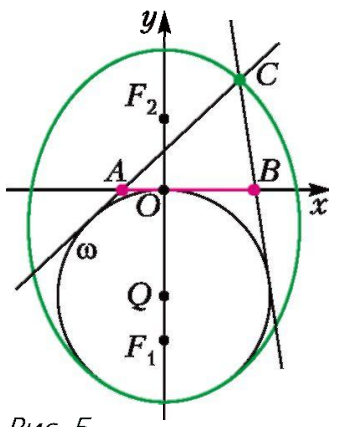
деляется как $\frac{\sqrt{a^{2}-\bar{y}^{\prime}}}{a}$. Тогда из формул (4) нетрудно увидеть, что отношение $a / R$, обозначенное нами через $e$, и есть әксцентриситет эллипса.

Упражнение 3. Проведите выкладку, показывающую, что $e=a / R$. (Не забудьте, что эллипс (3) вытянут по оси $O y$.)

3. Гипербола $(a>R)$.

У равнение (2) принимает вид

$$
-\frac{x^{2}}{p^{2}}+\frac{\left(y-y_{0}\right)^{2}}{q^{2}}=1
$$






$p=\frac{R^{2}}{v_{i 2^{2}} \quad R^{2}} \cdot q=\frac{R^{3}}{a^{2}-R^{2}}$,
$b_{: 1}=\frac{R\left(R^{2}-2 a^{2}\right)}{R^{2}-a^{2}}$. (5)

Фокусы гиперболы (рис.6) имеют координаты

$$
\begin{aligned}
& \left.F_{1} \mid 0 ;-R \frac{2 a+R}{R+a}\right)- \text { внутри } \omega, \\
& F_{2}\left(0 ; R \frac{2 a-R}{R-a}\right)-\text { всегда снаружи } \omega .
\end{aligned}
$$

Упражнение 4. Убедитесь в правильности приведенных формул. Проведите выкладку, показывающую, что әксцентриситет равен $e=a / R$ и в случае гиперболы. (Используйте тот факт, что для гиперболы, заданной каноническим уравнением $\frac{x^{2}}{a^{2}}-\frac{y^{2}}{b^{2}}=1$, где $a>0$, $b>0$, эксцентриситет определяется как $\frac{y^{i} a^{2}+b^{2}}{a}$.)

Попробуем подвести итоги нашей работы.

- Мы получили интересную параметризацию конических сечений дробно-рациональными функциями, при этом параметры имеют ясный геометрический смысл.

- Строго говоря, мы нашли не ГМТ, а лишь множество, содержащее это ГМТ. Но из простых геометрических соображений легко понять, что надо исключить лишь одну точку - вершину кривой, в которой происходит ее касание с окружно- стью ж. Эта точка соответствует «бесконечно удаленному» положению отрезка.

- Что объединяет все три случая? Одна конструкция, которая при различных соотношениях двух исходных параметров $R$ и $a$ определяет три конических сечения.

- Эксцентриситетом во всех случаях является отношение $a / R$, которое мы обозначили $e$. Это очевидно для параболы и нетрудно проверить в случаях эллипса и гиперболы, исходя из соотношений (4) и (5), определяющих длины полуосей этих кривых.

- Также нетрудно понять, что основная окружность $\omega$ касается кривой в ее вершине, причем радиус кривизны построенного конического сечения равен в этой вершине $R$.

- В рассматриваемой конструкции (при заданнњг $a$ и $R$ ) нетрудно построить циркулем и линейкой фокусы, директрисы, полуоси, асимптоты (для гиперболы) нашего ГМТ.

- Полученные результаты можно переформулировать и следующим образом. Построим окружность, вписанную в коническое сечение, касающуюся его в вершине и радиус которой равен радиусу кривизны кривой в этой точке. Затем проведем касательную к окружности в точке, диаметрально противоположной выбранной вершине, и на этой касательной расположим отрезок длиной, равной произведению диаметра окружности на эксцентриситет кривой. Тогда касательные к окружности в концах отрезка пересекутся на этой кривой.

Говорят, что у любого содержательного математического утверждения есть содержательный обратный факт. Давайте попробуем «развернуть» конструкцию. Представим себе, что отрезок $A B$ неподвижен, а окружность $\omega$ перемещается, оставаясь касательной к прямой $l$ (содержащей $A B$ ). В остальном сценарий останется прежним: из точек $A$ и $B$ проводим касательные к $\omega$, находим точку их пересечения $C$ и определяем ГМТ при всевозможных положениях окружности. Что әто будет за кривая? На первый взгляд, ничего существенно не изменилось, мы просто (выражаясь физическим языком) сменили систему отсчета. Но не торопитесь с выводами. Для экспериментов прекрасно подходит среда Geogebra. Дерзайте! 